\title{
Efficacy of Android Based Mobile Device as a Screening Tool for Hearing Loss in Quiet and Noisy Environments
}

\author{
Devendra Kumar Gupta ${ }^{1}$, Seema Pati², Sanajeet Singh³ ${ }^{3}$ Ravi Roy ${ }^{4}$, Rajeev Chugh ${ }^{5}$, Sunil Goyal ${ }^{6}$, Shivali Bansal7 , \\ Keerti Sangwan 8 \\ ${ }^{1}$ Associate Professor, Department of ENT- HNS, MS ENT, MCh, AHRR, New Delhi, India. \\ ${ }^{2}$ Junior Resident Department of ENT- HNS, AHRR, New Delhi, India. \\ ${ }^{3}$ Professor \& HOD, Department of ENT- HNS, AHRR, New Delhi, India. \\ ${ }^{4}$ Associate Professor, Department of ENT- HNS, MS ENT, AHRR, New Delhi, India. \\ ${ }^{5}$ Associate Professor, Department of ENT- HNS, MS ENT, AHRR, New Delhi, India. \\ ${ }^{6}$ Associate Professor, Department of ENT- HNS, MS ENT, AHRR, New Delhi, India. \\ ${ }^{7}$ Junior Resident, Department of ENT- HNS, AHRR, New Delhi, India. \\ 8Junior Resident, Department of ENT- HNS, AHRR, New Delhi, India.
}

\section{ABSTRACT}

\section{BACKGROUND}

Hearing loss has become an important global healthcare burden due to its high prevalence and its long-term implications on affected persons, communities and society as a whole. Developing countries like India has a high burden of hearing loss without an adequate infrastructure to screen and treat the disability. Hearing screening services scarcely extend to rural communities. To prevent the adverse effects of untreated hearing impairment, early identification through screening is important and advocated. Telehealth approaches have been utilized in recent years for hearing assessment and has gradually gained acceptance. With the advent of android based mobile technology, many applications are now available for screening hearing loss. However, their utility as an effective tool needs to be validated. The objective of this study is to determine the hearing levels of normal hearing and hearing impaired using an android based device, compare with that of the gold standard, Pure Tone Audiometry and validate the accuracy of new technique for hearing screening.

\section{METHODS}

33 participants (66 ears) with or without hearing impairment were examined for hearing threshold with a proprietary android based ENTraview device in sound treated as well as open environments and the results were compared with pure tone audiometer as the gold standard.

\section{RESULTS}

The test showed sensitivity of $96 \%$ and specificity of $82 \%$ of android device in sound treated room and $93 \%$ and $64 \%$ in noisy environment.

\section{CONCLUSIONS}

Android based ENTraview is a potential screening tool for early detection of hearing loss, which is accurate, reproducible and cheaper than PTA with the additional advantage of domiciliary testing. Health worker can use this application to screen people in community health center and if they fail screening, further referral to higher center can be recommended.

\section{KEY WORDS}

Audiometry, Pure Tone Audiometry, Hearing App

\author{
Corresponding Author: \\ Dr. Seema Pati, \\ L2/42B, First Floor \\ Kalkaji DDA Flats, \\ New Delhi-110019, \\ India. \\ E-mail: seema.cims@gmail.com \\ DOI: $10.14260 /$ jemds/2019/561 \\ Financial or Other Competing Interests: \\ None. \\ How to Cite This Article: \\ Gupta DK, Pati S, Singh S, et al. Efficacy of \\ android based mobile device as a screening \\ tool for hearing loss in quiet and noisy \\ environments. J. Evolution Med. Dent. Sci. \\ 2019;8(32):2577-2581, \\ DOI: \\ $10.14260 /$ jemds/2019/561
}

Submission 24-06-2019,

Peer Review 29-07-2019,

Acceptance 01-08-2019,

Published 12-08-2019. 


\section{BACKGROUND}

World over, 360 million suffer from disabling hearing loss, as estimated by WHO, making it the most common sensory impairment in humans.[1] $91 \%$ of these are in adults and the rest in children.[1] Disabling hearing loss in persons over 65 years affect around one third of population. Disabling hearing loss refers to hearing loss greater than $40 \mathrm{Db}$ in the better hearing ear in adults and greater than $30 \mathrm{~dB}$ in the better hearing ear in children. South Asia, Asia pacific and Sub Saharan Africa has got the highest prevalence of disabling hearing loss in both children and adults. One of the important strategies in hearing conservation is early detection especially regarding language development in children and cognitive decline in elderly. Early detection forms the key stone strategy for hearing rehabilitation as delay would lead to delay in speech and language development in children and cognitive decline in elderly. The impact of hearing loss on mental health and economic independence is substantial. The loss in the productivity of an individual due to hearing loss leads to high societal burden in terms of costs. If hearing impairment is identified early and treatment is provided, many of these negative effects can be avoided. Screening for hearing impairment can be useful for a range of age groups and patient groups, including newborns, to detect congenital hearing impairment; school children, to detect late -onset hearing impairment; the elderly, to identify age-related hearing loss (Presbycusis) \& those with HIV. However, substantial challenges exist in screening for hearing impairment such as the need for a quiet testing environment, prohibitively expensive specialist hearing assessment equipment that requires regular calibration\& skilled professional to conduct clinical tests. Highly sensitive tools that yield lower falsepositive and higher true negative results are ideal for screening and monitoring hearing. The conventional pure tone audiogram is considered the gold standard test to determine hearing handicap. For subjects less than 5 years objective tests like Otoacoustic emission and Brainstem Evoked Auditory Response are recommended. However, Pure Tone Audiometry has financial and logistic obstacles limiting its utility as a screening tool, especially in developing countries. ENTraview, an android based device has been developed as a screening modality for hearing loss with a view to be used in areas which are either remote or where the resources are limited. If proven to be sensitive in comparison to the gold standard Pure Tone Audiometry, [2] it could become a screening tool for detecting hearing impairment. By validating this device as a screening tool, primary care healthcare workers can detect hearing loss which can lead to reduction in unnecessary referrals to higher center. In view of this we conducted a preliminary study to evaluate the efficacy of device compare to Pure Tone Audiometry. The study purpose was to monitor the hearing for frequencies from $250 \mathrm{~Hz}$ to $8000 \mathrm{~Hz}$ of a nonrandomized heterogenous group consisting of normal hearing and abnormal hearing participants using an android based device in a sound treated room and in noisy environment and to compare both results. Both these methods were compared with Pure Tone Audiometry which is a gold standard test for assessment of hearing recognized by ASHA (American SpeechLanguage-Hearing Association) and BSA (British Society of Audiology).

The objective of the study was (a) To find a screening tool for early detection of hearing loss which is accurate and cheaper than Pure tone audiometry. (b) To validate the accuracy of new technique for hearing screening.

\section{METHODS}

The study was designed as a prospective observational intrasubject comparative study to assess the diagnostic accuracy of a hearing screening test. A mixed group consisting of both normally hearing and hearing loss individuals were part of this study. The study was approved by ethical committee of the institution. The participants were recruited from those attending the ENT out-patients department.

\section{Study Setting}

The study was conducted in the Department of Head and Neck Oncology, AHRR, Delhi Cant., India.

\section{Participants}

Sample size was calculated by taking confidence level of $95 \%$ and margin of error $1 \%$. Since this was a pilot study and we did not have an older data, variance was not known. 33 individuals participated in this study voluntarily. Informed Consent form was signed by all participants. The participation exclusion criteria were the following: Unwilling, 18 years of age or above and not able to perform the test. Participants were recruited from ENT outpatient department and otoscopic examination was performed on each participant, then they were sent for conventional audiometry examinations. The audiometry was performed by an audiologist using pure tone audiometry. The participants were then sent to quiet room where the android based device application was performed using the same procedure as for the typical audiometer when the atmosphere ambient sound measured approximately 30-40 dB. Valid testing was allowed by modern standards using these ambient sound levels. The criterion for abnormal hearing was predefined for the study which is $40 \mathrm{~dB}$ (Disabling hearing loss).

These patients were then subjected to hearing assessment by three independent assessors by three methods, namely-

a. The first assessor, a trained audiologist, conducted PTA using a calibrated audiometer (GSI 61 Clinical Audio meter and TDH 39P Headphones) in a sound treated room for frequencies ranging from $250 \mathrm{~Hz}$ to $8000 \mathrm{~Hz}$ using modified Hughson-Westlake procedure of bracketing. The pure tone audiometer was calibrated in decibels hearing level ( $\mathrm{Db} \mathrm{HL}$ ), according to the International Organization for Standardization. [3]

b. The second assessor, a trained nursing staff, coordinated the self-assessment of the hearing of the subject Android based device ENTraview (Manufactured by Medtronic) with a bundled headphone. It has customized software application and internet connectivity enabled operations, backup and storage of the data. It tests both ears individually \& sequentially the second assessor explained the steps of operation of the device to the subject. The subject, then, had to follow the instructions and operate the device on its touch screen interface. The test was performed in a sound treated environment, from low frequency to high frequency by default. The result was stored in tabulated as well as graphic form. 
c. How the device works-

Characteristic of android based app used by ENTraviewWith propriety software, the ENTraview is a standardized, CE certified, custom designed android phone that enables community health workers to store and share patient symptoms, treatment history, preliminary diagnosis and tympanic membrane images with health care providers for final diagnosis and treatment. Internet connectivity enables backup and central storage of data. It is rugged and portable for easy use in field. It has got rechargeable batteries. Audiometry application studies focus on comparison of only air conduction thresholds; thus, the fact is that they can be used as screening tools rather than diagnostic tools. Conductive and Sensorineural hearing loss can be differentiated from each other by Bone conduction testing. Further investigations are warranted for the validity of bone conduction testing. The utilization of headphones with greater attenuation of atmospheric ambient sound may improve the accuracy. If this is not possible, some attenuation from ambient noise may be provided by non-audiometric supra-aural headphones.

d. The third assessor coordinated the performance of the test with ENTraview in a non-sound treated environment using the identical procedure.

All audiometric data generated were compiled and statistical data analysis was done with an appropriate software. Typical Audiometric graph seen on android based ENTraview device.

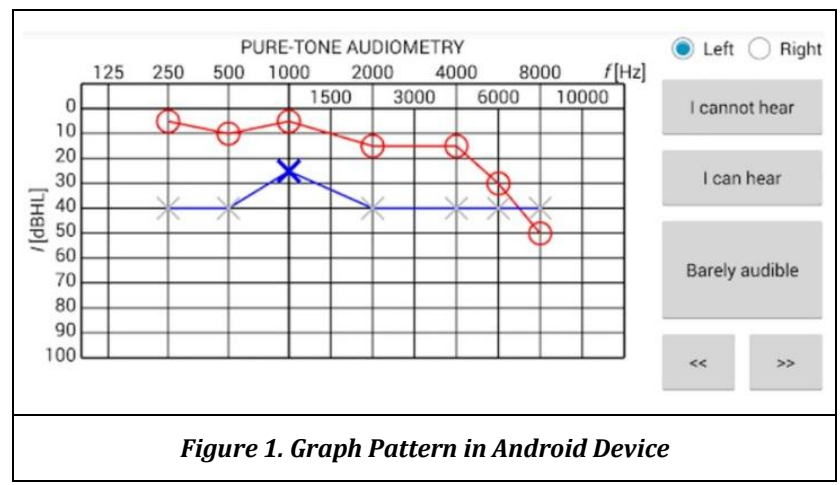

\section{Statistical Analysis}

Statistical analysis was done by using latest SPSS software. Receiver Operating Characteristic (ROC) was used as a method to compare the results and to know about Specificity, Sensitivity, Positive Predictive value and Negative Predictive Value.

\section{Ethics Committee Approval}

Institutional ethical committee approval was obtained for the study.

\section{RESULTS}

Out of 66 ears tested, 27 had hearing loss and 39 had normal hearing (Table 2). On comparison of air conduction thresholds for four frequencies $(500,1000,2000 \& 4000 \mathrm{~Hz})$ by PTA with identical frequencies of ENTraview in a sound treated environment, sensitivity was found to be $96 \%$ (Table $2 \& 3$ ) and specificity $57.5 \%$. On comparison of four frequencies (500, $1000,2000 \& 4000 \mathrm{~Hz}$ ) by PTA in a sound treated environment with that of ENTraview in a non-sound treated environment sensitivity was $93 \%$ and specificity was $64 \%$ (Table $4 \& 5$ ).The ROC table of both the comparison given in Table $3 \&$ Table 5.

\begin{tabular}{|c|c|c|c|c|c|c|c|}
\hline 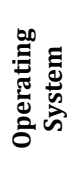 & 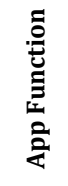 & $\overrightarrow{\tilde{s}}$ & 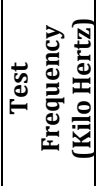 & 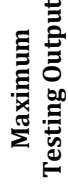 & 氖 & 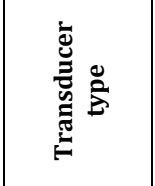 & 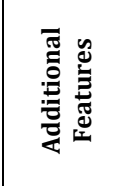 \\
\hline 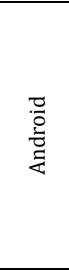 & 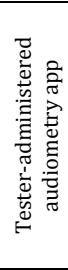 & 苋 & 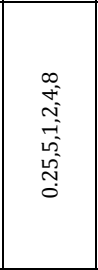 & $\begin{array}{l}\text { ?ै } \\
\text { ᄋ }\end{array}$ & \begin{tabular}{|} 
Calibrated with \\
non- \\
audiometric \\
headphones \\
according to \\
ISO389-1- \\
specified \\
standards \\
(within 0.1 Db \\
of accuracy
\end{tabular} & \begin{tabular}{|} 
Air- \\
conduction, \\
normal \\
headphones. \\
Bone \\
conduction not \\
measured
\end{tabular} & $\begin{array}{c}\text { Data } \\
\text { storage, } \\
\text { wifi } \\
\text { connectivity, } \\
\text { Otoscopy }\end{array}$ \\
\hline \multicolumn{8}{|c|}{ Table 1. Characteristics of Android Device ENTraview } \\
\hline
\end{tabular}

\begin{tabular}{|c|c|c|c|}
\hline Ear (RT/LT) & (All) & & \\
\hline Count of Patients & Android Device & & \\
\hline Office PTA & Yes & No & Grand Total \\
\hline Yes & 26 & 1 & 27 \\
\hline No & 32 & 7 & 39 \\
\hline Grand Total & 33 & 33 & 66 \\
\hline Table 2. Office PTA Vs Android Device (In Sound Treated Room) \\
\hline
\end{tabular}

\begin{tabular}{|c|c|}
\hline Receiver Operating Characteristic & \\
\hline True Positive (TP) & 26 \\
\hline True Negative (TN) & 32 \\
\hline False Positive (FP) & 7 \\
\hline False Negative (FN) & 1 \\
\hline & $96 \%$ \\
\hline Sensitivity (TP/(TP+FN)) & $82 \%$ \\
\hline Specificity (TN/(TN+FP)) & $79 \%$ \\
\hline PPV (TP/(TP+FP)) & $97 \%$ \\
\hline NPV (TN/(TN+FN)) & $88 \%$ \\
\hline & $87 \%$ \\
\hline Table 3. ROC of PTA Vs Android Device (In Sound Treated Room) \\
\hline
\end{tabular}

\begin{tabular}{|c|c|c|c|}
\hline Ear (RT/LT) & (All) & & \\
\hline Count of Patients & Android Devices & & \\
\hline Office PTA & Yes & No & Grand Total \\
\hline Yes & 25 & 2 & 27 \\
\hline No & 14 & 25 & 39 \\
\hline Grand Total & 39 & 27 & 66 \\
\hline Table 4. Office PTA Vs Android Devices (NE) \\
\hline
\end{tabular}

\begin{tabular}{|c|c|}
\hline Receiver Operating Characteristic & \\
\hline True positive (TP) & 25 \\
\hline True negative (TN) & 25 \\
\hline False Positive (FP) & 14 \\
\hline False Negative (FN) & 2 \\
\hline & $93 \%$ \\
\hline Sensitivity (TP/(TP+FN)) & $64 \%$ \\
\hline Specificity (TN/(TN+FP)) & $64 \%$ \\
\hline PPV (TP/(TP+FP)) & $93 \%$ \\
\hline NPV (TN/(TN+FN)) & \\
\hline & $76 \%$ \\
\hline Accuracy (TP+TN)/(TP+TN+FP+FN) & $76 \%$ \\
\hline F1 Score $2 \mathrm{TP} /(2 \mathrm{TP}+\mathrm{FP}+\mathrm{FN})$ & \\
\hline Table 5. ROC of PTA Vs Android Device (NE) & \\
\hline
\end{tabular}

\section{DISCUSSION}

Hearing impairment screening is not feasible for many people in developing countries due to constrain in resources, lack of skilled professionals not easily available to perform the required audiological tests \& high costs of audiological 
equipment. Hearing loss should be notice as early as possible and in order to ensure this hearing should be check from time to time especially in individuals with higher risk factors for hearing loss. Extensive review of literature using PubMed and Medline conducted in search of a quick, inexpensive and accurate method for detecting hearing loss. The wide distribution and mobility of smart phones make them an ideal platform for a variety of uses including tele health. A lot of recent publications have reported on the successful use of smart phone application in audiology. ${ }^{[4,5]}$ Some studies have suggested their enhanced utility with internet-based hearing test.[6,7,8] Also, there are studies indicating the use of smartphone-based hearing aid applications for providing temporary assistance to individuals with hearing deficits. ${ }^{\left[{ }^{9]}\right.}$ Android based applications has been used in several health screening instances. This include cardiovascular screening among others. Testing environment had a significant impact on the accuracy of results which have been demonstrated by several studies.[10,11,12] This finding is important, as ambient noise level in screening environments is a substantial challenge and can often exceed the recommended level of minimum of $40 \mathrm{~dB} .{ }^{[13]}$ Studies of audiometry app focused on comparison with air conduction thresholds only, thereby reinforcing that these applications function as screening tools rather than diagnostic tools. It does not suggest an alternative scenario to fully replacing audiometry. A recent study by Paglialongaa, Tognola and Pinciroli (2015) indicated that, in hearing health care, there is a distribution of available apps in five major categories; (i) education \& information (23\%) (ii) Hearing testing (18\%) (iii) Rehabilitation (24\%) (iv) SOUND enhancement (28\%) and (v) assistive tools (7\%)

High sensitivity of this tool across various sound environments suggests its potential as a good screening tool. This, in addition, to the ease of use and minimal cost makes it viable to be used on a large volume of subjects with ease in a short period of time. Access to audiometry being minimal in rural India, it gives the option of obtaining valid audiometer data with minimal cost on infrastructure and manpower. The smartphone is ubiquitous in India today and its geographical footprint makes this utility a useful tool for hearing evaluation in far flung and remote areas. Thus, it has the potential to become the game changer in hearing evaluation in primary health care and in monitoring in situations where there is a potential occupational noise hazard. The practical application of the smartphone as a screening tool for hearing in the field should not require the use of soundproof booths, and keeping with this all hearing test was carried out in noisy environment as well.

Application hearing tests are not without limitations regarding their performance. First the users can drive it to any result they want because of self-administration of the test. The instructions are in English language. Ambient noise should be prevented therefore the test must be done in a quiet room. The android application uses pure tones which is less reliable than speech audiometry which can be performed by trained audiologists. The hearing test cannot not differentiate between conductive and sensorineural hearing loss which is important to communities in underserved areas. There may be inaccurate results, particularly for low frequencies, because of the ambient noise. Calibration issues may also affect the results obtained. While the audiometer is routinely calibrated, the developers of the smartphone application do not directly calibrate the earphones bundled with the phones but provide an automatic download of the calibration coefficients for the specific model for the android device with its bundled earphones, the implication of this is that replacing/ interchanging the ear-phones means a recalibration is required before the results can be valid. Currently active research is ongoing introducing the technology of active noise reduction into audiometry headphones or earphones which will allow for more accurate measurements of hearing in the field despite background noise.

\section{Recommendation}

The author recommends this device to be used for Screening, Ototoxicity monitoring, Occupational monitoring where no formal audiology available. In settings where there is full access to proper audiological investigations it can be used for screening purpose.

\section{CONCLUSIONS}

For early detection of hearing loss in underserved areas, smart phone applications are good alternative tests with low-cost solutions. It is easy to perform and can be done by an individual at any time. In the past few years, android applications have gained much interest for hearing loss detection and screening. However, much work needs to be done for validation of the technology and to develop it as a means of delivering services as the field is in its infancy. It is our moral responsibility and obligation to penetrate the undeserved localities with audiological services as there is absence of proper hearing health care for majority of people with impaired hearing globally. This device looks very promising for bridging this gap by providing services through the ever expanding reach of global connectivity.

\section{REFERENCES}

[1] Mortality and Burden of Diseases and prevention of Blindness and Deafness WHO, 2012. http://www.who.int/pbd/deafness/WHO_GE-HL.pdf

[2] Recommended procedure for pure-tone bone-conduction audiometry without masking using a manually operated instrument. British Society of Audiology--technical note. Br J Audiol 1985;19(4):281-2.

[3] International Organization for Standardization Technical report (1998). Acoustics-Reference zero for the calibration of audiometric equipment. Part 1- Reference equivalent threshold sound pressure levels for pure tones and supra-aural earphones. ISO/TR 389-1.

[4] De Swanepoel W, Myburgh HC, Howe DM, et al. Smartphone hearing screening with integrated quality control and data management. International Journal of Audiology 2014;53(12):841-9.

[5] Honeth L, Bexelius C, Eriksson M, et al. An internet-based hearing test for simple audiometry in nonclinical settings: preliminary validationand proof of principle. Otology \& Neuroautology 2010;31(5):708-14. 
[6] Na Y, Joo HS, Yang H, et al. Smartphone-based hearing screening in noisy environments. Sensors (Basel) 2014;14(6):10346-60.

[7] Bexelius C, Honeth L, Ekman A, et al. Evaluation of an internet-based hearing test-comparison with established methods for detection of hearing loss. J Med Internet Res 2008;10(4):e32.

[8] Nast DR, Speer WS, Le Prell CG. Sound level measurements using smartphone "apps": useful or inaccurate? Noise Health 2014;16(72):251-6.

[9] Amlani A, Taylor B, Levy C, et al. Utility of smartphonebased hearing aid applications as a substitute to traditional hearing aids. Hearing Review 2013;12:16-23.
[10] Szudek J, Ostevik A, Dziegielewski P, et al. Can Uhear me now? Validation of an iPod-based hearing loss screening test. J Otolaryngol Head Neck Surg 2012;41 Suppl 1:S7884.

[11] Foulad A, Bui P, Djalilian H. Automated audiometry using apple IOS-based application technology. Otolaryngol Head Neck Surg 2013;149(5):700-6.

[12] Peer S, Fagan JJ. Hearing loss in the developing world: evaluating the iPhone mobile device as a screening tool. S Afr Med J 2015;105(1):35-9.

[13] Arlinger S. Negative consequences of uncorrected hearing loss - a review. Int J Audiol 2003;42 Suppl 2:2S17-20. 